\title{
Nonlinear Transfer Function-Based Image Detail Preserving Dynamic Range Compression for Color Image Enhancement
}

\author{
Deepak Ghimire and Joonwhoan Lee \\ Computer Science and Engineering, Chonbuk National University, \\ Jeonju 561-756, Jeollabuk-do, Rep. of Korea \\ \{deep, chlee\}@chonbuk.ac.kr
}

\begin{abstract}
This paper presents a method for color image enhancement in HSV space with preserving image details. The RGB color image is converted into HSV space and V channel image is now subjected for enhancement. By applying image dependent nonlinear transfer function the local image contrast preserving dynamic range compression as well as contrast enhancement is performed simultaneously on the V channel image. Finally, the enhanced V channel image and original $\mathrm{H}$ and $\mathrm{S}$ channel images are converted back to RGB image to obtain enhanced RGB image. The original color of the image is preserved because $\mathrm{H}$ and $\mathrm{S}$ component are kept unchanged. The experimental results show that the performance of the proposed method is better in terms of both subjective and objective evaluation in comparison with conventional methods.
\end{abstract}

Keywords: nonlinear transfer function, dynamic range compression, image local contrast, multiscale enhancement.

\section{Introduction}

The image captured in natural environment with high dynamic range (HDR) includes both bright and dark regions. The camera has the capability to capture high dynamic range images, while most of the display devices have low dynamic range. On the other hand if the dynamic range of human eye sensing is exceeded, it is difficult to perceive the HDR images. Image enhancement with dynamic range compression is a common approach to improve the quality of those images in terms of human visual perception. The image enhancement techniques can be divided into two categories namely: spatial domain methods and transform domain methods. In spatial domain methods the intensity of the pixel in image is directly manipulated. But in transform domain techniques the image intensity data is transformed into specific domain by using methods such as DFT, DCT, DWT, etc. and the frequency content of the image is altered for image enhancement.

Various image processing technique have been developed to improve the quality of the image in terms of human visual perception. One of the traditional and well known techniques for image contrast enhancement is histogram equalization (HE). But $\mathrm{HE}$ in its original form tends to introduce some annoying artifacts and unnatural enhancement. To overcome those problems different variants of traditional HE method are developed. 
In the literature methods like [1], [2], [3], [4] etc. can be found as modified HE based method for image contrast enhancement. Methods like [1], [2] divides image into two sub images based on mean and median of the original image respectively and performs the $\mathrm{HE}$ in each image independently. At last, the processed sub images are composed into one image to get the final result. An improved version of those methods is presented in [3]. Here separation is done recursively; separates each new histogram further based on their respective mean. As the number of separation increases, the output image's mean brightness will converge to the input image's mean brightness. Similarly method [4] modifies the histogram of the original image by weighting and thresholding before the HE. But, the problem with HE based methods is that it is indiscriminate. It may increase the contrast of background noise, while decreasing the usable signal. On the other hand it produces unrealistic effects in the images.

R. Fattel et al. [5] developed a gradient domain high dynamic range compression method. They modified the gradient field of the luminance image by attenuating magnitude of the large gradients and obtain the low dynamic range image by solving a Poisson equation on the modified gradient field. On the other hand Debevec et al. [6] develop a method of recovering high dynamic range radiance maps from ordinary photographs. Their algorithm used the multiple differently exposed photographs to recover the response function of the imaging process and with this response function the algorithm can fuse the multiple photographs into single, high dynamic range radiance map. The multiscale retinex based method, e.g., Jobson et al. [7], and single scale retinex based image enhancement method, e.g., Choi et al. [8] are also developed, in which luminance enhancement and contrast enhancement are realized simultaneously. Tao et al. [9] presented an adaptive and integrated neighborhood dependent approach for nonlinear enhancement (AINDANE) to improve the visual quality of digital images captured under low or nonuniform illumination conditions. It consists of two independent processes: adaptive luminance enhancement and adaptive contrast enhancement, which are applied to treat luminance information of images. Recently, many transform based enhancement techniques have also been developed. Xiao et al. [10] proposed a method for enhancing contrast of the image based on wavelet transform and human visual system. Clement et al. [11] developed the image enhancement algorithm in compressed DCT domain which is able to enhance both dark and bright region of an image equally well.

Most of the digital video cameras have adopted a knee curve as a dynamic range compression function. This method strongly compressed the highlighted range over the knee point, so the contrast in the highlighted region is much degraded. To improve this problem an auto knee curve has been used for dynamic range compression. However, the auto knee slightly improves the highlight contrast instead of lowering the luminance in the middle range. To solve these problems an approximated auto knee curve is used by Monobe et al. [12] for dynamic range compression with preserving local image contrast. This algorithm automatically and adaptively enhances the local image contrast in the highlighted regions. Here, because the use of approximated auto knee curve local contrast capabilities are limited. On the other hand, we would like to able to have strong enhancement capability and like to extend the enhancement in the middle and low frequency regions. To achieve this goal, in this paper we are using the concept of contrast preserving proposed by [12] with nonlinear transfer function as dynamic range compression function. In this paper the 
concepts from [9] and [12] are combined to achieve color image enhancement in HSV space. Image enhancement in HSV space has the advantage of preserving color and saturation of the image by only modifying value component of the original image in the enhancement process. Recently, D. Ghimire et al. [13] presented a method for image enhancement in HSV space by considering image locality in dynamic range compression process. This is the improved version of the method proposed in [9]. In this paper we are also using the concept of multiscale image convolution to improve the result of image enhancement.

The rest of the paper is organized as follows. In section 2, the procedure of image enhancement in HSV space is presented. In section 3, experimental results along with performance evaluation of the proposed method are shown. Finally the conclusion is given in section 4 .

\section{Local Contrast Preserving Image Enhancement}

In general, color images are represented in RGB color space. This paper uses HSV color space for image enhancement, in which the hue $(H)$ refers to the spectral composition of color, saturation $(S)$ defines the purity of colors and value $(V)$ refers the brightness of a color or just the luminance value of the color. Here RGB values of the image are converted into HSV values and then the value component image is now subjected for enhancement. HSV color space is selected in this image enhancement procedure to preserve the saturation and color of the input image.

In this paper we are using the basic concept of local contrast preserving proposed in [12] for dynamic range compression. The mathematical condition to preserve the local contrast is described as follows.

$$
\frac{g(x, y)}{g_{\text {ave }}(x, y)}=\frac{f(x, y)}{f_{\text {ave }}(x, y)}
$$

where, $f(x, y)$ and $f_{\text {ave }}(x, y)$ denotes the input luminance level and the input local average, $g(x, y)$ and $g_{\text {ave }}(x, y)$ denotes the output luminance level and the output local average of each pixel $(x, y)$ of the value component image in HSV space respectively.

The core equation describing the condition to preserve the local contrast in dynamic range compression process given by [12] is described as follows.

$$
\begin{gathered}
g(x, y)=p(f(x, y)) \times r\left(f(x, y), f_{\text {ave }}(x, y)\right) \\
r\left(f(x, y), f_{\text {ave }}(x, y)\right)=\left(\frac{f(x, y)}{f_{\text {ave }}(x, y)}\right)^{\alpha\left\{1-\frac{f(x, y)}{p(f(x, y))} \frac{d p(f(x, y))}{d f(x, y)}\right\}}
\end{gathered}
$$

where, $p(f(x, y))$ denotes an arbitrary tone mapping curve in luminance domain, and $\alpha$ denotes a gain parameter for the local contrast enhancement.

The local average $f_{\text {ave }}(x, y)$ in (2) and (3) is calculated by taking the convolution of spatial averaging filter $A(x, y)$ and the input value component image $f(x, y)$ as follows,

$$
f_{\text {ave }}(x, y)=A(x, y) \otimes f(x, y)
$$


Here, the 2-D Gaussian function $A(x, y)$ is defined as

$$
A(x, y)=K \exp \left[\frac{-\left(x^{2}+y^{2}\right)}{\sigma^{2}}\right]
$$

The standard deviation $(\sigma)$ of the 2-D Gaussian distribution determines the size of the neighborhood. In this equation $K$ is a gain factor and is determined by

$$
\iint K \exp \left[\frac{-\left(x^{2}+y^{2}\right)}{\sigma^{2}}\right] d x d y=1
$$

The selection of tone mapping function, $p(f(x, y))$ in Eq. (2) and (3) is very important and will affect the result of enhancement directly. In [12], the authors are using approximated knee curve as a tone mapping curve. But the knee curve only compresses the highlighted range over the knee point. On the other hand here we want the strong enhancement capabilities and like to extend the enhancement in the middle and low frequency regions too. Different type of tone mapping function can be used to have different type of enhancement results. In this paper we are trying to enhance the regions with low intensity or with dark pixels. Therefore, we have selected the nonlinear transfer function as mapping function used by [9] for luminance enhancement which also serves as dynamic range compression and is defined as

$$
p(f(x, y))=\frac{f(x, y)^{(0.75 z+0.25)}+0.5(1-f(x, y))(1-z)+f(x, y)^{(2-z)}}{2}
$$

The range of $f(x, y)$ in Eq. (7) is 0 to 1 . The nonlinear transfer function provided in Eq. (7) is image dependent with parameter $z$ and is calculated by using following relation

$$
z=\left\{\begin{array}{cl}
0 & \text { for } L \leq 50 \\
\frac{L-50}{100} & \text { for } 50<L \leq 150 \\
1 & \text { for } L>150
\end{array}\right.
$$

where $L$ is the luminance level corresponding to a cumulative distribution function (CDF) of 0.1 of value component input image in HSV space. The range of $L$ is 0 to 255.

The image dependent parameter $z$ can be calculated from the image globally, or can be calculated from the value component image locally. Calculating parameter locally depending upon each small region of the image and applying luminance enhancement with different shaped transfer function for each corresponding small region can produce better result than applying global transfer function in luminance enhancement process [13]. But, calculating parameter locally increases the computational complexicity of the algorithm. Fig. 1 shows the shape of nonlinear transfer function for different values of $z$ and Fig. 2 shows an input value component image along with corresponding CDF. 


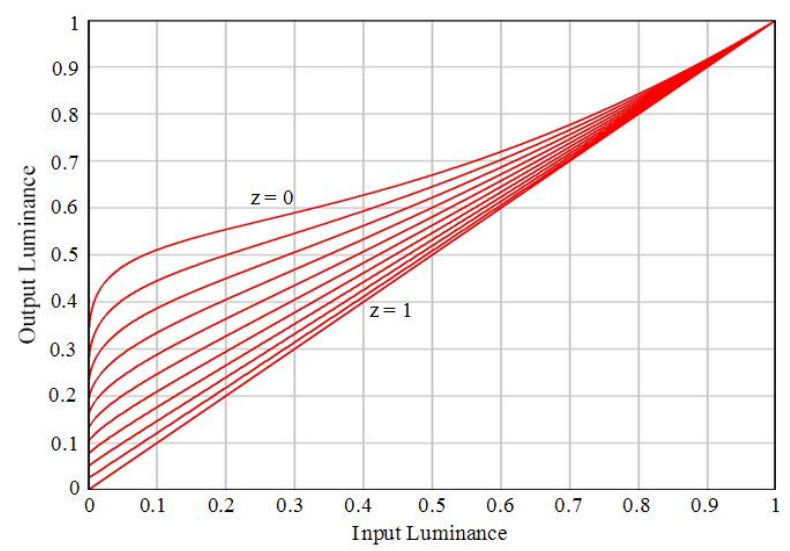

Fig. 1. Shape of nonlinear transfer functions with different $\mathrm{z}$ values
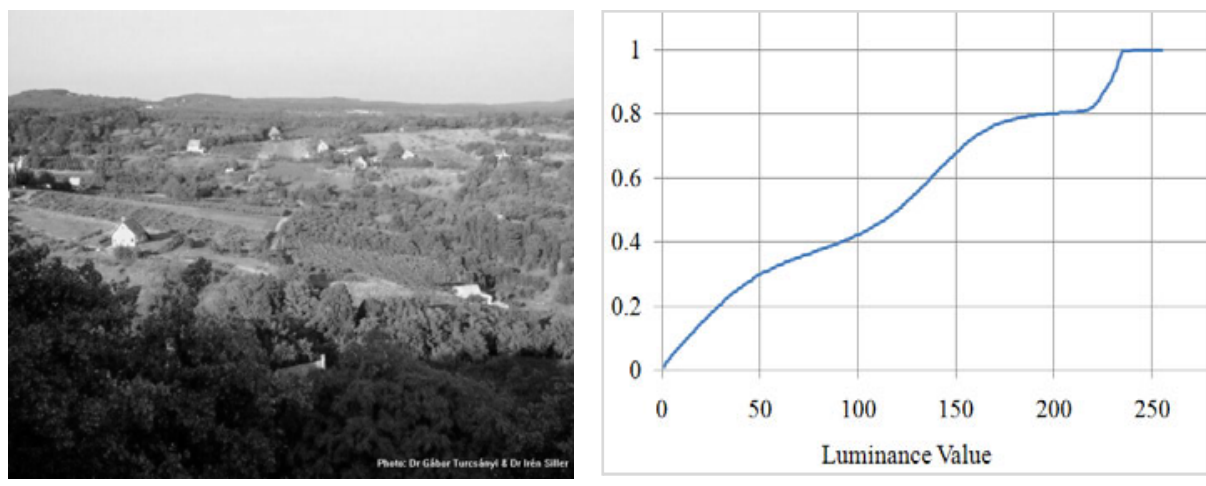

Fig. 2. An intensity image and its cumulative distribution function (CDF)

According to Eq. (3) we need differential function of nonlinear transfer function of Eq. (7) which is defined as

$$
\frac{d p(f(x, y))}{d f(x, y)}=\frac{(0.75 z+0.25) f(x, y)^{(0.75 z-0.75)}+0.5(z-1)+(2-z) f(x, y)^{(1-z)}}{2}
$$

Now Eq. (3) can be applied for image enhancement. In our experiment we are using value of $\alpha$ as 1.5 , because we want to increase the local contrast of the input image. Selection of scale (standard deviation) in Gaussian function is another important aspect in image enhancement procedure because it directly affects the result of enhancement. Convolution with a small scale, such as a few neighboring pixels, can provide luminance information about the nearest neighborhood pixels, while the convolution with a large scale comparable to the image dimensions can provide the 
information about the large-scale luminance variation over the whole image. Generally, smaller scale convolution tends to produce result with fine details and convolution with larger scale tends to produce natural looking and smooth results. A medium scale convolution can produce combination of both small scale and large scale results. Therefore we can use multiple scale convolutions to produce different results and we can combine all of them to find the final image enhancement result. But, if we need faster processing we can use medium scale convolution in image enhancement process. On the other hand we can also use parallel processing to find the enhancement results in different scales to get rid from the computational complexicity. The image enhancement with multiscale convolution can be described by the following equations

$$
\begin{gathered}
g_{i}(x, y)=p(f(x, y)) \times r\left(f(x, y), f_{\text {ave }, i}(x, y)\right) \\
r\left(f(x, y), f_{\text {ave }, i}(x, y)\right)=\left(\frac{f(x, y)}{f_{\text {ave }, i}(x, y)}\right)^{\alpha\left\{1-\frac{f(x, y)}{p(f(x, y))} \frac{d p(f(x, y))}{d f(x, y)}\right\}} \\
f_{\text {ave }, i}(x, y)=A_{i}(x, y) \otimes f(x, y) \\
A_{i}(x, y)=K \exp \left[\frac{-\left(x^{2}+y^{2}\right)}{\sigma_{i}^{2}}\right] \\
g(x, y)=\frac{\sum_{i=1}^{n} g_{i}(x, y)}{n}
\end{gathered}
$$

where $n$ is the number of scales and $\sigma_{i}$ represents different scales.

In our experiment, we selected $n=3$ and find the enhanced images in three scales: small, medium and large. In this work, used three scales are 5, 15 and 50. It is experimentally determined that those scales are suitable for almost all type of images. After obtaining the average enhancement result of value component image in HSV space by using Eq. (14), it is combined with original $H$ and $S$ component images and converted back to RGB space to find the final result in RGB space. The saturation and hue channels are not altered in this image enhancement procedure.

\section{Results and Discussion}

The proposed algorithm has been applied to large number of digital images captured under dark illumination conditions for performance evaluation and comparison with other techniques. This section contains some results as well as discussion about the performance of the proposed algorithm for image enhancement. The proposed 
algorithm is also compared with other methods using both subjective and objective evaluation.

\subsection{Subjective Evaluation}

Image enhanced with various scales in Gaussian function are shown in Fig. 3. The effect of different scales can be clearly seen in these enhanced images. Result of image enhancement using very small scale has the richer local details and result by using large sale has smoothing effect. Convolution with medium scale has the result in somewhere middle of both large and small scale result. Therefore, from this result it is clear that combination of all the scales can produce better results. But the computational complexicity is increased in multiscale convolution image enhancement process if computation is carried out in serial manner.

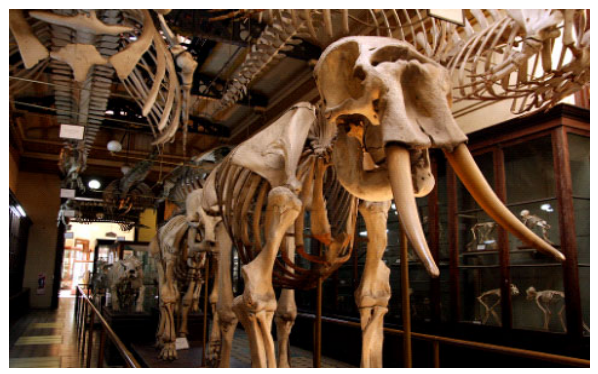

(a)

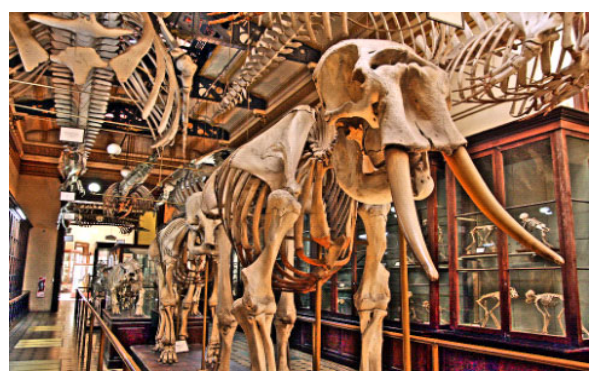

(c)

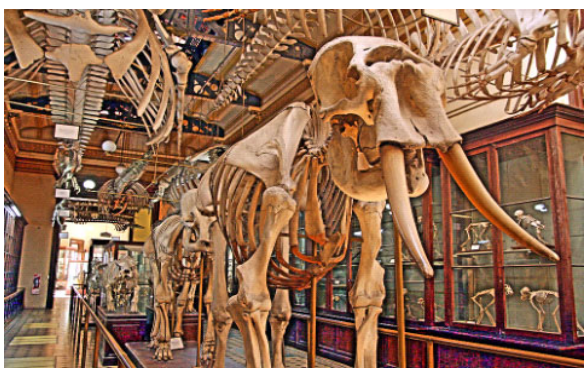

(b)

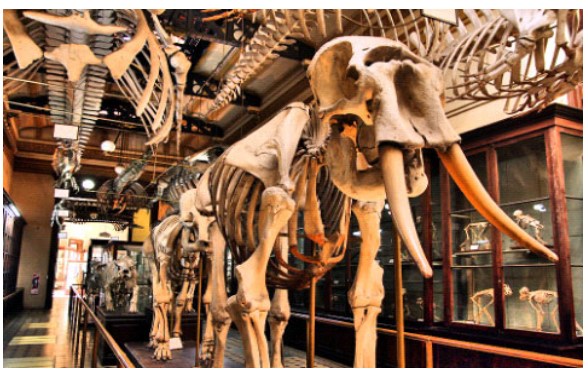

(d)

Fig. 3. Image enhancement results with different scales in Gaussian function: (a) Input image, (b) enhancement using scale $=5$, (c) enhancement using scale $=10$, and (d) enhancement using scale $=50$

Here we compare the performance of the proposed method with the HE, AINDANE [9], MSRCR [7] and literature [13]. The enhanced image using proposed method has fine local and global details with natural looking, and balanced luminance and contrast across the whole image and no change in original color of the image in 
comparison with other methods. Fig. 4 shows two input color images and result of enhancement using different methods. The color of the image is changed as well as the local contrast of the image is increased unnecessarily using MSRCR method. The global enhancement using AINDANE and [13] are satisfactory, but local details are still to be enhanced. In the output image of our method both local and global contrast are increased well with preserving image details. Here we are using miltiscale convolution for enhancement using proposed method. More results of image enhancement using proposed method are shown in Fig. 5. The experiment on other test images has shown similar results.
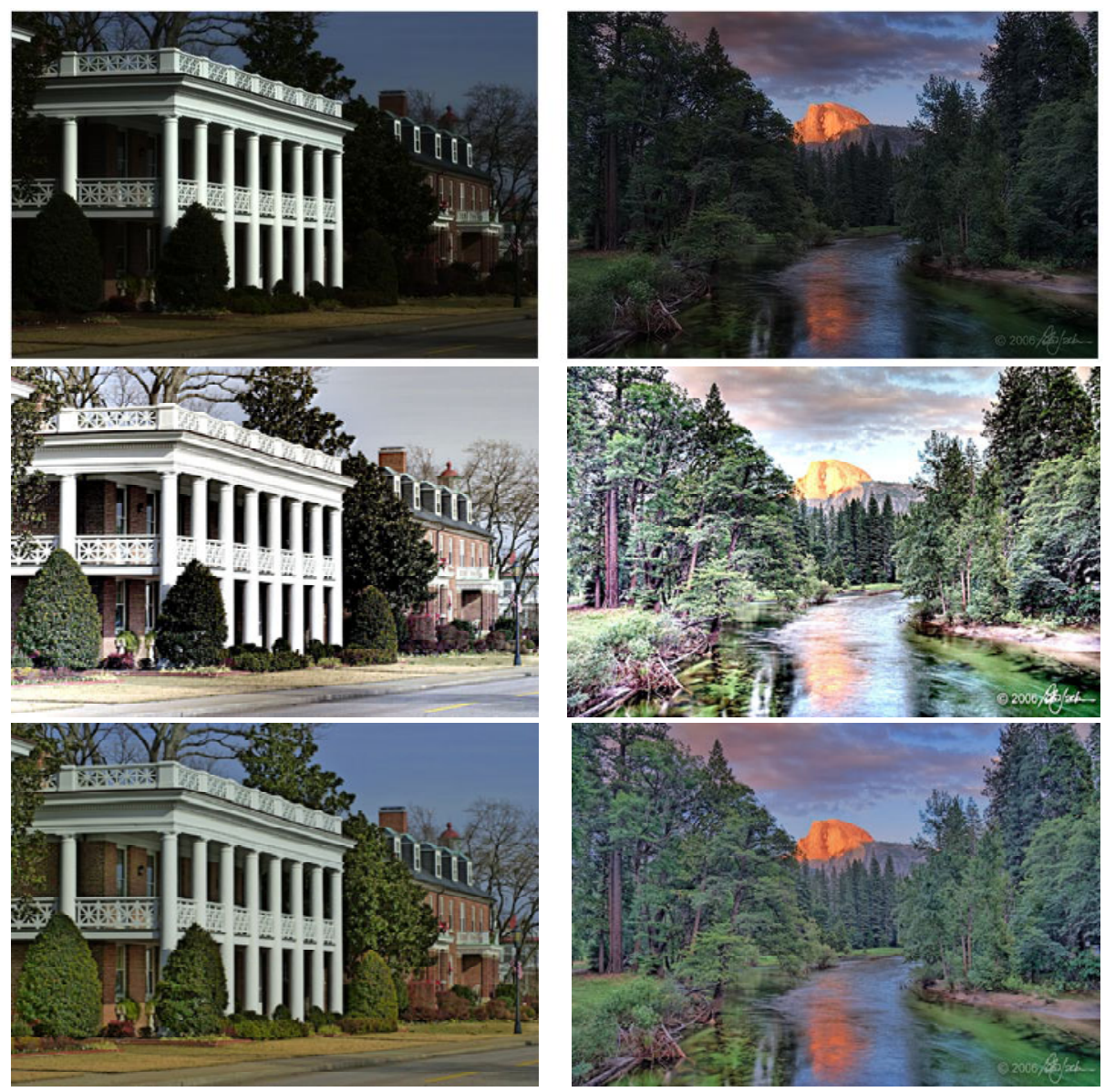

Fig. 4. Comparison of image enhancement with different methods: Input color images (first row), enhancement results using MSRCR (second row), enhancement results using AINDANE (third row), enhancement results using [13] (fourth row) and enhancement results using proposed method (last row) 

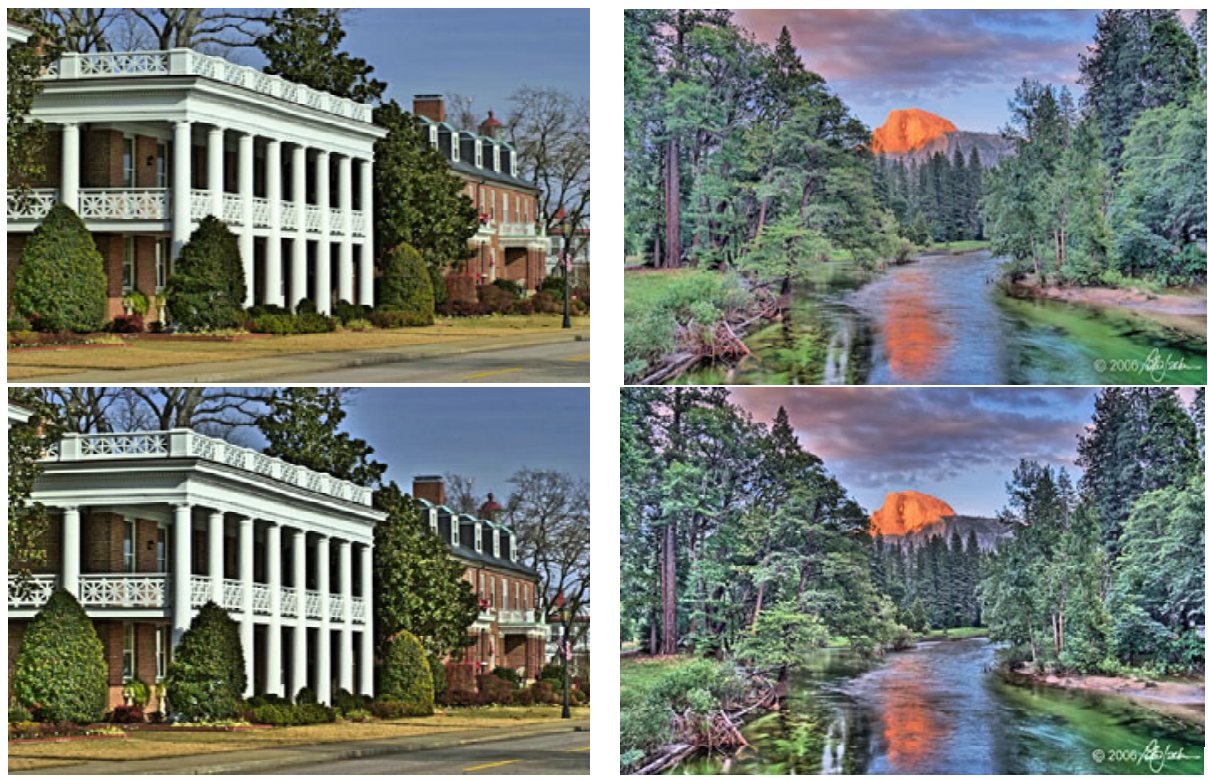

Fig. 4. (continued)
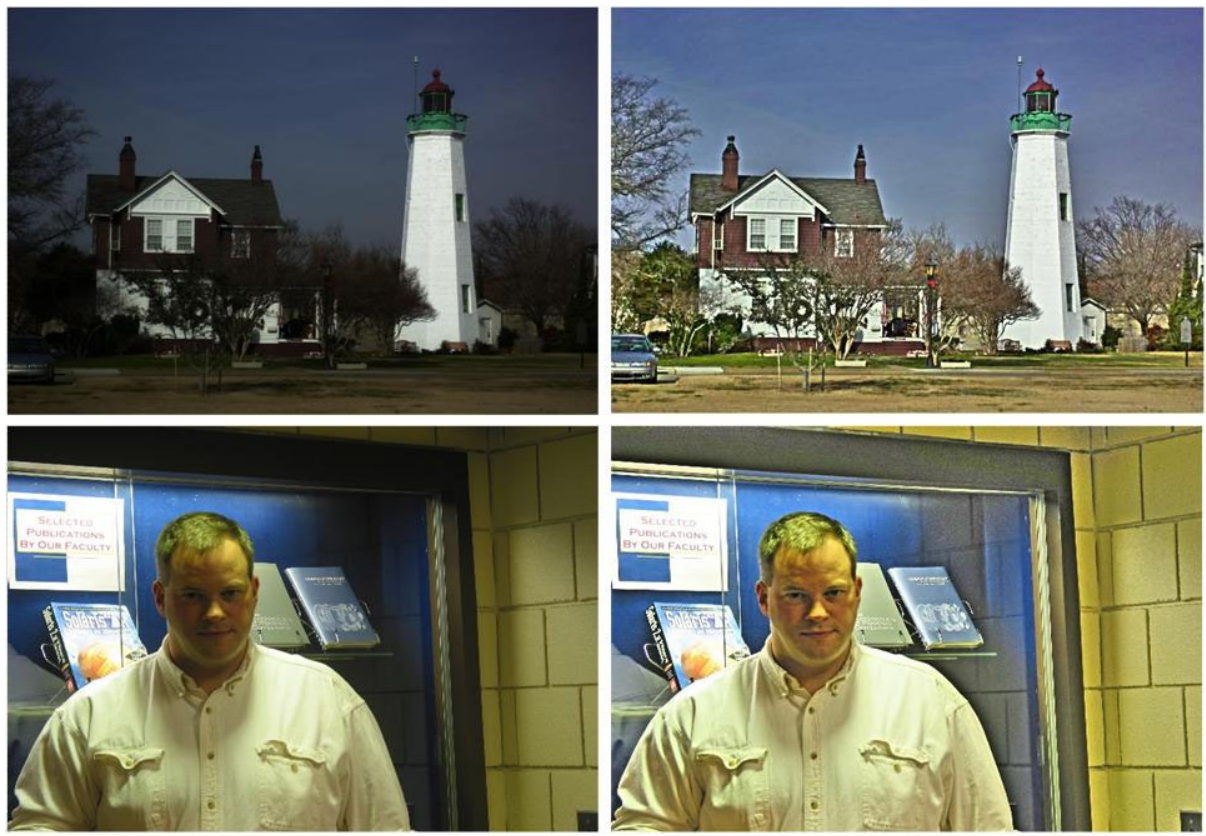

Fig. 5. More results of image enhancement using proposed method 


\subsection{Objective Evaluation}

In this subsection, we use the objective evaluation criteria to compare the performance of the proposed method with other methods. One of the objective evaluation criteria was taken to be the Detail Variance (DV) and Background Variance (BV) from [14]. DV and BV values are obtained firstly by computing the variance of the gray-levels in the neighboring pixels of each pixel in the image. After that, the pixel is classified to the foreground when the variance is more than a threshold; otherwise the pixel is classified to the background. DV is the average variance of the pixels included in the detail region, and BV is the average variance of all the pixels included in the background region. The desired result is increase in DV and no change in BV after applying the enhancement [14]. Here the size of the neighborhood is chosen to be $7 \times 7$ and threshold was chosen to be 5 . Results for some test images are shown in Table 1. From Table 1 it is clear that the results are better than AINDANE and Literature [13], and are comparable to the MSRCR. The variance of the detail region is increased sufficiently and variance of the background region is unchanged, which is desirable. Even the result of MSRCR seems best, from the subjective evaluation it is clear that MSRCR produces unnatural result with unnecessarily increase in contrast.

The proposed image enhancement method is also compared with other methods by using statistical method proposed by D. J. Jabson et al. [15]. In this method, the statistical properties of image, mean, and the mean of zonal standard deviation, are used to describe the visual quality of the image in terms of image contrast and details. Here, first we divide the image into $40 \times 40$ non overlapping pixel blocks and for each block mean and standard deviation is calculated and plotted as shown in Fig. 6 with different enhancement results. The image quality is classified as visually optimal if it lies inside the white rectangle region [15]. The blue data points indicates the position of the small block of the original image and corresponding red data points connected via a straight line indicates the position of those small blocks of the image after enhancement. Fig. 6 (a) shows the enhanced image using MSRCR and corresponding statistical plot of that image, Fig. 6 (b) shows the enhanced image using AINDANE and corresponding statistical plot and Fig. 6 (c) shows the enhanced image using proposed method and corresponding statistical plot. By using proposed method $50 \%$ of the red points are inside the white rectangle where as only $32 \%$ and $47 \%$ of the red points is inside white rectangle using AINDANE and MSRCR enhancement methods respectively. This proves the robustness of the proposed method in comparison with other methods.

Table 1. DV and BV values for different enhancement results

\begin{tabular}{|c|c|c|c|c|c|c|c|c|c|c|}
\hline \multirow{2}{*}{ Image } & \multicolumn{2}{|c|}{ Original } & \multicolumn{2}{c|}{ MSRCR [7] } & \multicolumn{2}{c|}{$\begin{array}{c}\text { AINDANE } \\
{[9]}\end{array}$} & \multicolumn{2}{c|}{$\begin{array}{c}\text { Literature } \\
{[\mathbf{1 3}]}\end{array}$} & \multicolumn{2}{c|}{$\begin{array}{c}\text { Proposed } \\
\text { Method }\end{array}$} \\
\cline { 2 - 11 } & BV & DV & BV & DV & BV & DV & BV & DV & BV & DV \\
\hline 1 & 1.52 & 15.03 & 1.50 & 35.80 & 1.09 & 17.37 & 1.23 & 21.10 & 1.21 & 28.59 \\
\hline 2 & 2.80 & 23.95 & 2.83 & 41.60 & 3.39 & 26.11 & 3.47 & 36.38 & 3.47 & 36.38 \\
\hline 3 & 3.27 & 25.18 & 3.21 & 57.40 & 3.54 & 35.87 & 3.47 & 40.78 & 3.50 & 54.30 \\
\hline 4 & 2.48 & 21.56 & 1.27 & 38.91 & 1.82 & 24.15 & 1.92 & 28.09 & 1.69 & 39.30 \\
\hline Average & $\mathbf{2 . 5 1}$ & $\mathbf{2 1 . 4 3}$ & $\mathbf{2 . 2 0}$ & $\mathbf{4 3 . 4 2}$ & $\mathbf{2 . 4 6}$ & $\mathbf{2 5 . 8 7}$ & $\mathbf{2 . 5 2}$ & $\mathbf{3 1 . 5 8}$ & $\mathbf{2 . 4 6}$ & $\mathbf{3 9 . 6 4}$ \\
\hline
\end{tabular}



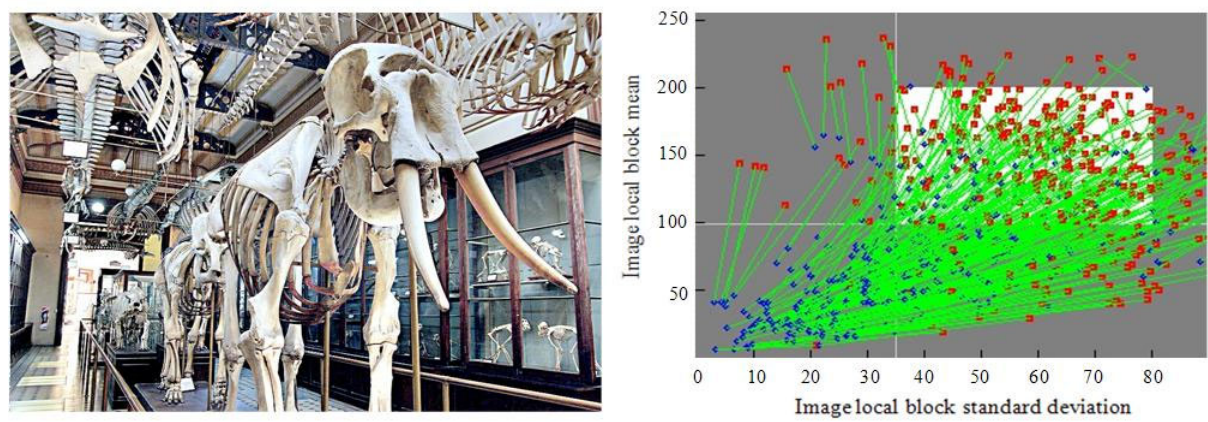

(a)
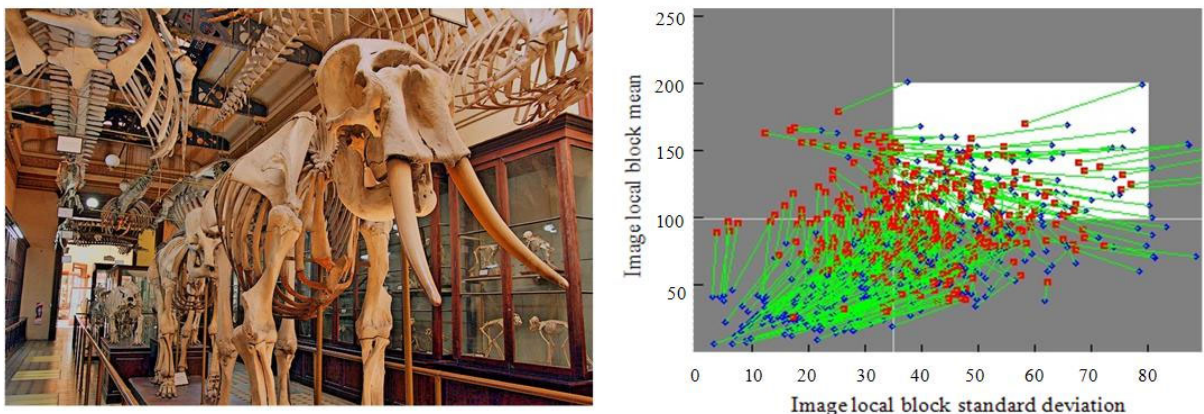

(b)
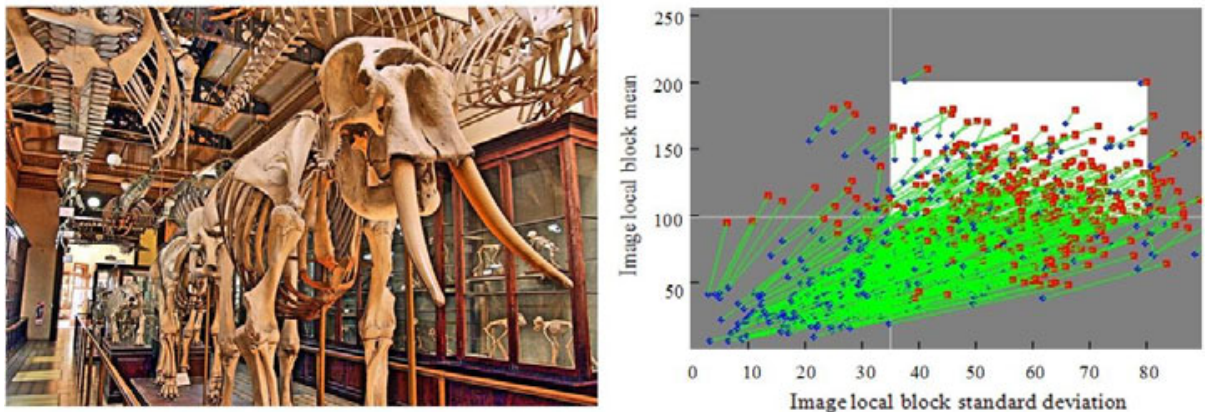

(c)

Fig. 6. Enhancement results with statistical characteristics of image of Fig. 3 (a) (image local block mean versus corresponding local block standard deviation) using: (a) MSRCR method, (b) AINDANE method, and (c) proposed method

\section{Conclusion}

In this paper we propose a color image enhancement method in HSV space with preserving image details. Both subjective and objective performance evaluation has shown the proposed method is superior then other enhancement methods like 
MSRCR, AINDANE and [13]. The enhancement results are natural with no change in original color, because the use of HSV space for image enhancement without changing hue and saturation of the input image. The future work will focus on decreasing computational complexicity and making the enhancement results more natural looking without degrading quality of enhancement results.

\section{References}

1. Kim, Y.-T.: Contrast Enhancement Using Brightness Preserving Hi-Histogram Equalization. IEEE Trans. on Consumer Electronics 43, 1-8 (1997)

2. Wang, Y., Chen, Q., Zhang, B.: Image Enhancement Based on Equal Area Dualistic SubImage Histogram Equalization Method. IEEE Trans. on Consumer Electronics 45, 68-75 (1999)

3. Chen, S.-D., Ramli, R.: Contrast Enhancement using Recursive Mean-Separate Histogram Equalization for Scalable Brightness Preservation. IEEE Trans. on Consumer Electronics 49, 1301-1309 (2003)

4. Wang, Q., Ward, R.K.: Fast Image/Video Contrast Enhancement Based on Weighted Threshold Histogram Equalization. IEEE Trans. on Consumer Electronics 53, 757-764 (2007)

5. Fattal, R., Lischinski, D., Werman, M.: Gradient Domain High Dynamic Range Compression. In: Proc. of the 24th Annual Conference on Computer Graphics and Interactive Technologies, pp. 249-256 (2002)

6. Debevec, P.E., Malik, J.: Recovering High Dynamic Range Radiance Maps from Photographs. In: Proc. of the 24th Annual Conference on Computer Graphics and Interactive Technologies, pp. 369-378 (1997)

7. Jobson, D.J., Rahman, Z., Woodell, G.A.: A Multiscale Retinex for Bridging the Gap between Color Images and the Human Observation of Scenes. IEEE Trans. on Image Processing 6, 965-976 (1997)

8. Choi, D.H., Jang, I.H., Kim, M.H., Kim, N.C.: Color Image Enhancement using SingleScale Retinex based on an Improved Image Formation Model. In: 16th Ruropean Conf. (EUSIPCO 2008), Lausanne, Switzerland (2008)

9. Tao, L., Asari, V. K.: Adaptive and Integrated Neighborhood-Dependent approach for Nonlinear Enhancement of Color Images. J. of Electronic Imaging 14, 043006-1-04300614 (2005)

10. Xiao, D., Ohya, J.: Contrast Enhancement of Color Images Based on Wavelet Transform and Human Visual System. In: Proc. of the IASTED Int. Conf. Graphics and Visualization in Engg., Florida, USA, pp. 58-63 (2007)

11. Clement, J.C., Parbukumar, M., Baskar, A.: Color Image Enhancement in Compressed DCT Domain. ICGST-GVIP Journal 10, 31-38 (2010) ISSN: 1687-398X

12. Monobe, Y., Yamashita, H., Kurosawa, T., Kotora, H.: Dynamic Range Compression Preserving Local Image Contrast for Digital Video Camera. IEEE Trans. on Consumer Electronics 51, 1-10 (2005)

13. Ghimire, D., Lee, J.: Nonlinear Transfer Function-Based Local Approach for Color Image Enhancement. IEEE Trans. on Consumer Electronics 57, 858-865 (2011)

14. Ramponi, G., Strobel, N.K., Mitra, S.K., Yu, T.-H.: Nonlinear Unsharp Masking Methods for Image Contrast Enhancement. J. of Electronic Imaging 5, 353-366 (1996)

15. Jobson, D.J., Rahman, Z., Woodell, G.A.: Statistic of Visual Representation. In: Proc. of SPIE, vol. 4736, pp. 25-35 (2002) 